\title{
Double field theory as the double copy of Yang-Mills theory
}

\author{
Felipe Díaz-Jaramillo๑, ${ }^{*}$ Olaf Hohm, ${ }^{\dagger}$ and Jan Plefka ${ }^{\star}$ \\ Institut für Physik und IRIS Adlershof, Humboldt-Universität zu Berlin, \\ Zum Großen Windkanal 2, 12489 Berlin, Germany
}

(Received 15 September 2021; accepted 7 February 2022; published 17 February 2022)

\begin{abstract}
We show that double field theory arises from the color-kinematic double copy of Yang-Mills theory. A precise double copy prescription for the Yang-Mills action at quadratic and cubic order is provided that yields the double field theory action in which the duality invariant dilaton has been integrated out. More precisely, at quadratic order this yields the gauge-invariant double field theory, while at cubic order it yields the cubic double field theory action subject to a gauge condition that originates from Siegel gauge in string field theory.
\end{abstract}

DOI: 10.1103/PhysRevD.105.045012

\section{INTRODUCTION}

The fundamental interactions in nature, as far as currently known, are governed by two kinds of theories: YangMills theory, which describes the gauge bosons of the standard model of particle physics, and Einstein's theory of general relativity, which describes the force of gravity and the universe at large. While these two kinds of theories share broad qualitative features, such as the presence of gauge symmetries, their actions appear to be quite different. Most importantly, at the quantum field theory level, the perturbative expansion of general relativity about flat space-time is notoriously involved and nonrenormalizable, in sharp contrast to the well-understood perturbation theory of Yang-Mills theory.

Yet, the Bern-Carrasco-Johansson construction [1,2] of gravity scattering amplitudes as the "double copy" of YangMills amplitudes suggests that there might be a much more intimate relationship between gauge theory and gravity. Specifically, the perturbation theory of Yang-Mills and more general gauge theories can be arranged in such a way that their building blocks obey a property known as colorkinematics duality. Here the contributions associated with the gauge group known as "color factors" appear on the same footing as the purely kinematical numerator factors (functions of momenta and polarizations), which in turn obey relations akin to Jacobi identities. Replacing the color

\footnotetext{
*felipe.diaz-jaramillo@hu-berlin.de †ohohm@physik.hu-berlin.de

jan.plefka@hu-berlin.de
}

Published by the American Physical Society under the terms of the Creative Commons Attribution 4.0 International license. Further distribution of this work must maintain attribution to the author(s) and the published article's title, journal citation, and DOI. Funded by SCOAP. factors by kinematic factors then yields gravity amplitudes, whose computation by standard textbook methods starting from the Einstein-Hilbert Lagrangian is incomparably more involved; see [3] for a recent review and [4] for a popular account. Somewhat misleadingly, these observations are often summarized by "gravity is Yang-Mills squared." Formal proofs of the double copy have been provided at tree level $[5,6]$ using a variety of methods. Evidence for the existence also at loop level has been provided through a number of explicit constructions; see [3] for an account and $[7,8]$ for a treatment in terms of homotopy algebras. (In addition, the double copy prescription has been extended to the sector of classical solutions [9].)

Double copy techniques are a main feature of the modern amplitude program, which largely abandons the textbook approach to quantum field theory with its baggage of offshell fields and gauge redundancies; see [10] for reviews. As such, there is no reason to expect that features of the double copy can be seen at the level of an action. To quote [11]: "no amount of fiddling with the Einstein-Hilbert action will reduce it to a square of a Yang-Mills action." Nevertheless, there have been numerous attempts to render the double copy at least partially manifest at the level of a Lagrangian $[5,12,13]$.

A first step is to rewrite the perturbative expansion of gravity in a way that manifests a left-right index factorization. Starting from the Einstein-Hilbert action this requires a series of elaborate field redefinitions that were worked out to a small order in fields in [12]. This problem is solved in the framework of double field theory [14-18] that doubles space-time yielding an automatic index factorization pertaining to the two space-time coordinates. Double field theory was originally conceived of as a nonlocal field theory, referred to as weakly constrained double field theory, that captures, by virtue of fields depending on doubled coordinates, massive Kaluza-Klein and winding modes of string 
theories on toroidal backgrounds [15]. While this theory has not yet been constructed explicitly beyond cubic order, much work has been done on strongly constrained double field theory, in which the doubling of coordinates is purely formal but which provides a duality covariant and background-independent reformulation of the target space actions of string theory including metric, B field, and dilaton. (See [19] for reviews and [20] for earlier important work.) It was anticipated by Siegel in [14] that these reformulations render index factorizations manifest, and this was established explicitly to all orders in fields in [21] and used in [22] for amplitude computations. (See also [23], where the problem of index factorizations was revisited more recently, and [24-26] for double copy prescriptions of classical solutions in double field theory.)

In this paper we go beyond this and ask: what does a double copy prescription applied to the Yang-Mills Lagrangian actually give? As the double copy instructs us to replace color factors with a second set of kinematic factors, which come with their own momenta, this naturally leads to a double field theory with doubled momenta or, in position space, a doubled set of coordinates. The key technical result reported here is that this double copy of Yang-Mills theory yields at quadratic and cubic order double field theory upon integrating out the duality invariant dilaton. ${ }^{1}$ More precisely, at quadratic order this match holds at the level of gauge-invariant Lagrangians, while at cubic order this match requires a gauge choice, which in the string field theory formulation of [15] originates from the so-called Siegel gauge and which reduces to the de Donder gauge for standard gravity fields.

\section{QUADRATIC THEORY}

We start from the action for Yang-Mills theory in $D$ dimensions,

$$
S_{\mathrm{YM}}=-\frac{1}{4} \int d^{D} x \kappa_{a b} F^{\mu \nu a} F_{\mu \nu}{ }^{b},
$$

with the field strength for the gauge bosons $A_{\mu}{ }^{a}$,

$$
F_{\mu \nu}{ }^{a}=\partial_{\mu} A_{\nu}{ }^{a}-\partial_{\nu} A_{\mu}{ }^{a}+g_{\mathrm{YM}} f_{b c}^{a} A_{\mu}{ }^{b} A_{\nu}{ }^{c},
$$

where $g_{\mathrm{YM}}$ is the gauge coupling. Here, $f_{b c}^{a}$ denotes the structure constants of the color gauge group, with adjoint indices $a, b, \ldots$, and invariant Cartan-Killing form $\kappa_{a b}$ that lowers adjoint indices, so that $f_{a b c} \equiv \kappa_{a d} f^{d}{ }_{b c}$ is totally antisymmetric. Expanding the Yang-Mills action to quadratic order in fields and integrating by parts, one obtains

\footnotetext{
${ }^{1}$ The duality invariant dilaton $\phi$ of double field theory is defined in terms of the familiar scalar dilaton $\Phi$ by $e^{-2 \phi}=$ $\sqrt{-g} e^{-2 \Phi}$.
}

$$
S_{\mathrm{YM}}^{(2)}=\frac{1}{2} \int d^{D} x \kappa_{a b} A^{\mu a}\left(\square A_{\mu}{ }^{b}-\partial_{\mu} \partial^{\nu} A_{\nu}{ }^{b}\right) .
$$

In order to motivate the double copy prescription it is convenient to pass over to momentum space. Defining $A_{\mu}{ }^{a}(k) \equiv \frac{1}{(2 \pi)^{D / 2}} \int d^{D} x A_{\mu}{ }^{a}(x) e^{i k x}$ the quadratic action reads

$$
S_{\mathrm{YM}}^{(2)}=-\frac{1}{2} \int_{k} \kappa_{a b} k^{2} \Pi^{\mu \nu}(k) A_{\mu}{ }^{a}(-k) A_{\nu}{ }^{b}(k),
$$

where $\int_{k}:=\int d^{D} k$, and we have scaled out $k^{2}$, in order to define the projector in terms of the Minkowski metric $\eta_{\mu \nu}=\operatorname{diag}(-,+,+,+)$ :

$$
\Pi^{\mu \nu}(k) \equiv \eta^{\mu \nu}-\frac{k^{\mu} k^{\nu}}{k^{2}},
$$

which obeys the identities

$$
\Pi^{\mu \nu}(k) k_{\nu} \equiv 0, \quad \Pi^{\mu \nu} \Pi_{\nu \rho} \equiv \Pi_{\rho}^{\mu} .
$$

The first identity implies gauge invariance under

$$
\delta A_{\mu}{ }^{a}(k)=k_{\mu} \lambda^{a}(k)
$$

where the gauge parameter $\lambda^{a}(k)$ is an arbitrary function.

Let us now turn to the double copy construction of a gravity theory. We take the double copy prescription to lead to a double field theory: Replace the color indices $a$ by a second set of space-time indices denoted by a bar, $a \rightarrow \bar{\mu}$, corresponding to a second set of space-time momenta $\bar{k}^{\bar{\mu}^{2}}$ :

$$
A_{\mu}{ }^{a}(k) \rightarrow e_{\mu \bar{\mu}}(k, \bar{k}) .
$$

To complete the double copy prescription for the quadratic theory we need to define a substitution rule for the CartanKilling metric $\kappa_{a b}$. We will see that the following replacement does the job:

$$
\kappa_{a b} \rightarrow \frac{1}{2} \bar{\Pi}^{\bar{\mu} \bar{\nu}}(\bar{k})
$$

where $\bar{\Pi}^{\bar{\mu}} \bar{\nu}$ is defined as in (5), but with all momenta replaced by barred momenta and all indices replaced by barred indices. This prescription is motivated from the double copy rule at the level of amplitudes: for a gauge theory amplitude $\mathcal{A}=\sum_{i} \frac{n_{i} c_{i}}{D_{i}}$, where $n_{i}$ are kinematic factors, $c_{i}$ are color factors, and the $D_{i}$ are the inverse

\footnotetext{
${ }^{2}$ The mass dimensions in momentum space are $[A]=-1-\frac{D}{2}$ and $[e]=-1-D$, so this substitution does not preserve the dimensions, which is due to the additional $\bar{k}$ integral. Similarly, the dimensions of the coupling constants are $\left[g_{\mathrm{YM}}\right]=2-\frac{D}{2}$ and $[\kappa]=1-D$ for the gravitational coupling in double field theory, which differs from that in Einstein gravity given by $1-\frac{D}{2}$.
} 
propagators, the double copy amounts to replacing $c_{i}$ by kinematic factors $n_{i}$, while the $D_{i} \sim k^{2}$ are untouched. Thus, it is natural to scale out $k^{2}$ from the kinetic operator and to double only the resulting projector $\Pi^{\mu \nu}$, which also guarantees that a two-derivative theory is mapped to a twoderivative theory.

The quadratic gravity action following from this double copy (DC) prescription then reads

$S_{\mathrm{DC}}^{(2)}=-\frac{1}{4} \int_{k, \bar{k}} k^{2} \Pi^{\mu \nu}(k) \bar{\Pi}^{\bar{\mu} \bar{\nu}}(\bar{k}) e_{\mu \bar{\mu}}(-k,-\bar{k}) e_{\nu \bar{\nu}}(k, \bar{k})$.

We emphasize that the field $e_{\mu \bar{\mu}}$ now depends on doubled momenta $K \equiv(k, \bar{k})$. Note that the momenta $k$ and $\bar{k}$ enter the action on the same footing, except that we have chosen the factor in front to be $k^{2}$ rather than $\bar{k}^{2}$, but in double field theory this asymmetry is resolved due to the so-called level-matching constraint [15]

$$
k^{2}=\bar{k}^{2},
$$

where two copies of the same flat space-time metric are used to take the square. In order to match with double field theory, and also to lead to a local action, we thus have to assume that the doubled momenta are subject to this constraint (which does have more general solutions than the trivial $k=\bar{k}$ for which the theory reduces to a standard linearized gravity theory). We also note that, owing to the first identity in (6), the action is manifestly gauge invariant under

$$
\delta e_{\mu \bar{\nu}}=k_{\mu} \bar{\lambda}_{\bar{\nu}}+\bar{k}_{\bar{\nu}} \lambda_{\mu}
$$

with two independent gauge parameters $\lambda_{\mu}$ and $\bar{\lambda}_{\bar{\mu}}$ that depend on doubled momenta $K \equiv(k, \bar{k})$, subject to (11).

We will now show that (10) is indeed equivalent to (quadratic) double field theory. Writing out the projectors with (5) and using the level-matching constraint (11) the action reads

$$
\begin{aligned}
S_{\mathrm{DC}}^{(2)}= & -\frac{1}{4} \int_{k, \bar{k}}\left(k^{2} e^{\mu \bar{\nu}} e_{\mu \bar{\nu}}-k^{\mu} k^{\rho} e_{\mu \bar{\nu}} e_{\rho}^{\bar{\nu}}-\bar{k}^{\bar{\nu}} \bar{k}^{\bar{\sigma}} e_{\mu \bar{\nu}} e^{\mu} \overline{\bar{\sigma}}\right. \\
& \left.+\frac{1}{k^{2}} k^{\mu} k^{\rho} \bar{k}^{\bar{\nu}} \bar{k}^{\bar{\sigma}} e_{\mu \bar{\nu}} e_{\rho \bar{\sigma}}\right) .
\end{aligned}
$$

In order to compare with the standard double field theory action we have to Fourier transform to (doubled) position space. This is straightforward except for the last term in (13), which due to the factor $\frac{1}{k^{2}}$ would yield a nonlocal term. This problem is resolved by introducing an auxiliary scalar field $\phi(k, \bar{k})$ (the dilaton):

$$
\begin{aligned}
S_{\mathrm{DC}}^{(2)}= & -\frac{1}{4} \int_{k, \bar{k}}\left(k^{2} e^{\mu \bar{\nu}} e_{\mu \bar{\nu}}-k^{\mu} k^{\rho} e_{\mu \bar{\nu}} e_{\rho}^{\bar{\nu}}-\bar{k}^{\bar{\nu}} \bar{k}^{\bar{\sigma}} e_{\mu \bar{\nu}} e^{\mu}{ }_{\bar{\sigma}}\right. \\
& \left.-k^{2} \phi^{2}+2 \phi k^{\mu} \bar{k}^{\bar{\nu}} e_{\mu \bar{\nu}}\right) .
\end{aligned}
$$

Integrating out $\phi$ by solving its own field equations,

$$
\phi=\frac{1}{k^{2}} k^{\mu} \bar{k}^{\bar{\nu}} e_{\mu \bar{\nu}},
$$

and backsubstituting into the action we recover the nonlocal (13). Alternatively, without integrating out fields, one may redefine the dilaton as $\phi \rightarrow \phi^{\prime}=\phi-\frac{1}{k^{2}} k^{\mu} \bar{k}^{\bar{\nu}} e_{\mu \bar{\nu}}$, which decouples $\phi^{\prime}$ from $e_{\mu \bar{\nu}}$. The action (14) is of course still gauge invariant, with a gauge transformation for $\phi$ that is determined by the variation of (15):

$$
\delta \phi=k_{\mu} \lambda^{\mu}+\bar{k}_{\bar{\mu}} \bar{\lambda}^{\bar{\mu}},
$$

where we used (11). With the action in the form (14) it is then straightforward to Fourier transform to a local action in doubled position space:

$$
\begin{aligned}
S_{\mathrm{DC}}^{(2)}= & \frac{1}{4} \int d^{D} x d^{D} \bar{x}\left(e^{\mu \bar{\nu}} \square e_{\mu \bar{\nu}}+\partial^{\mu} e_{\mu \bar{\nu}} \partial^{\rho} e_{\rho}^{\bar{\nu}}\right. \\
& \left.+\bar{\partial}^{\bar{\nu}} e_{\mu \bar{\nu}} \bar{\partial}^{\bar{\sigma}} e^{\mu}{ }_{\bar{\sigma}}-\phi \square \phi+2 \phi \partial^{\mu} \bar{\partial}^{\bar{\nu}} e_{\mu \bar{\nu}}\right),
\end{aligned}
$$

where $\partial_{\mu}=\frac{\partial}{\partial x^{\mu}}$ and $\bar{\partial}_{\bar{\mu}}=\frac{\partial}{\partial \bar{x}^{\bar{\mu}}}$ are the partial derivatives corresponding to the coordinates that are dual to $k^{\mu}$ and $\bar{k}^{\bar{\mu}}$ and hence by (11) subject to the constraint

$$
\square \equiv \partial^{\mu} \partial_{\mu}=\bar{\partial} \bar{\mu} \bar{\partial}_{\bar{\mu}}
$$

The gauge transformations (12) and (16) translate in doubled position space to

$$
\begin{aligned}
\delta e_{\mu \bar{\nu}} & =\partial_{\mu} \bar{\lambda}_{\bar{\nu}}+\bar{\partial}_{\bar{\nu}} \lambda_{\mu}, \\
\delta \phi & =\partial_{\mu} \lambda^{\mu}+\bar{\partial}_{\bar{\mu}} \bar{\lambda}^{\bar{\mu}},
\end{aligned}
$$

under which (17) is invariant, modulo the constraint (18). The action (17) defines precisely the standard quadratic double field theory action, which upon setting $x=\bar{x}$ is equivalent, up to field redefinitions, to the familiar free action for gravity, antisymmetric tensor and dilaton [15].

\section{CUBIC THEORY}

We now turn to the cubic vertex of Yang-Mills theory and extend the double copy construction to the cubic action of double field theory. The cubic part of the Yang-Mills action (1) reads

$$
S_{\mathrm{YM}}^{(3)}=-g_{\mathrm{YM}} \int d^{D} x f_{a b c} \partial^{\mu} A^{\nu a} A_{\mu}{ }^{b} A_{\nu}{ }^{c} .
$$


Upon Fourier transforming to momentum space this becomes

$S_{\mathrm{YM}}^{(3)}=\frac{i g_{\mathrm{YM}}}{(2 \pi)^{D / 2}} \int_{k_{1}, k_{2}, k_{3}} \delta\left(k_{1}+k_{2}+k_{3}\right) f_{a b c} k_{1}^{\mu} A_{1}^{\nu a} A_{2 \mu}{ }^{b} A_{3 \nu}{ }^{c}$,

where we use the shorthand notation $A_{i} \equiv A\left(k_{i}\right)$, and we performed the $x$ integration, introducing the delta function. It is convenient to write this more symmetrically as

$$
\begin{aligned}
S_{\mathrm{YM}}^{(3)}= & -\frac{i g_{\mathrm{YM}}}{6(2 \pi)^{D / 2}} \int_{k_{1}, k_{2}, k_{3}} \delta\left(k_{1}+k_{2}+k_{3}\right) \\
& \times f_{a b c} \Pi^{\mu \nu \rho}\left(k_{1}, k_{2}, k_{3}\right) A_{1 \mu}{ }^{a} A_{2 \nu}{ }^{b} A_{3 \rho}{ }^{c},
\end{aligned}
$$

where we defined

$$
\Pi^{\mu \nu \rho}\left(k_{1}, k_{2}, k_{3}\right) \equiv \eta^{\mu \nu} k_{12}^{\rho}+\eta^{\nu \rho} k_{23}^{\mu}+\eta^{\rho \mu} k_{31}^{\nu},
$$

with $k_{i j} \equiv k_{i}-k_{j}$. Note that this tensor has the antisymmetry properties required by the structure it multiplies, e.g., $\Pi^{\mu \nu \rho}\left(k_{1}, k_{2}, k_{3}\right)=-\Pi^{\nu \mu \rho}\left(k_{2}, k_{1}, k_{3}\right)$.

Our task now is to give the double copy prescription that extends (8), (9) to the cubic theory. The natural substitution rule is

$$
f_{a b c} \rightarrow \frac{i}{4} \bar{\Pi}^{\bar{\mu} \bar{\nu} \bar{\rho}}\left(\bar{k}_{1}, \bar{k}_{2}, \bar{k}_{3}\right),
$$

where the factor of $i$ is needed since we relate a theory with one derivative to a theory with two derivatives. Together with $g_{\mathrm{YM}} \rightarrow \frac{1}{2} \kappa$, after which we set $\kappa=1$, this gives the cubic action

$$
\begin{aligned}
S_{\mathrm{DC}}^{(3)}= & \frac{1}{48(2 \pi)^{D / 2}} \int d K_{1} d K_{2} d K_{3} \delta\left(K_{1}+K_{2}+K_{3}\right) \\
& \times \bar{\Pi}^{\bar{\mu} \bar{\nu} \bar{\rho}}\left(\bar{k}_{1}, \bar{k}_{2}, \bar{k}_{3}\right) \Pi^{\mu \nu \rho}\left(k_{1}, k_{2}, k_{3}\right) e_{1 \mu \bar{\mu}} e_{2 \nu \bar{\nu}} e_{3 \rho \bar{\rho}},
\end{aligned}
$$

where we use the shorthand notation $e_{i \mu \bar{\mu}} \equiv e_{\mu \bar{\mu}}\left(K_{i}\right)$, with $K \equiv(k, \bar{k})$ for doubled momenta, and $d K \equiv d^{2 D} K$. Writing out $\Pi^{\mu \nu \rho}$ and $\bar{\Pi}^{\bar{\mu} \bar{\nu} \bar{\rho}}$ yields nine terms which, upon relabeling momentum variables and indices, reduce to two terms, and then writing out $k_{i j}=k_{i}-k_{j}$ the action becomes

$$
\begin{aligned}
S_{\mathrm{DC}}^{(3)}= & \frac{1}{8(2 \pi)^{D / 2}} \int d K_{1} d K_{2} d K_{3} \delta\left(K_{1}+K_{2}+K_{3}\right) \\
& \times e_{1 \mu \bar{\mu}}\left[-k_{2}^{\mu} e_{2 \rho \rho \bar{\rho}} \bar{k}_{3}^{\bar{\mu}} e_{3}^{\rho \bar{\rho}}+k_{2}^{\mu} e_{2 \nu \bar{\rho}} \bar{k}_{3}^{\bar{\rho}} e_{3}^{\nu \bar{\mu}}+k_{2}^{\rho} e_{2}^{\mu \bar{\rho}} \bar{k}_{3}^{\bar{\mu}} e_{3 \rho \bar{\rho}}\right. \\
& \left.+k_{2}^{\mu} \bar{k}_{2}^{\bar{\mu}} e_{2 \rho \bar{\rho}} e_{3}^{\rho \bar{\rho}}-k_{2 \rho} e_{2}^{\mu \bar{\rho}} \bar{k}_{3 \bar{\rho}} e_{3}^{\rho \bar{\mu}}-k_{2}^{\rho} \bar{k}_{2}^{\bar{\mu}} e_{2}^{\mu \bar{\rho}} e_{3 \rho \bar{\rho}}\right] .
\end{aligned}
$$

Fourier transforming to position space and integrating by parts, we finally obtain

$$
\begin{aligned}
S_{\mathrm{DC}}^{(3)}= & \frac{1}{8} \int d^{D} x d^{D} \bar{x} e_{\mu \bar{\mu}}\left[2 \partial^{\mu} e_{\rho \bar{\rho}} \bar{\partial}^{\bar{\mu}} e^{\rho \bar{\rho}}-2 \partial^{\mu} e_{\nu \bar{\rho}} \bar{\partial}^{\bar{\rho}} e^{\nu \bar{\mu}}\right. \\
& \left.-2 \partial^{\rho} e^{\mu \bar{\rho}} \bar{\partial}^{\bar{\mu}} e_{\rho \bar{\rho}}+\partial^{\rho} e_{\rho \bar{\rho}} \bar{\partial}^{\bar{\rho}} e^{\mu \bar{\mu}}+\bar{\partial}_{\bar{\rho}} e^{\mu \bar{\rho}} \partial_{\rho} e^{\rho \bar{\mu}}\right] .
\end{aligned}
$$

In the following we will prove that this action agrees precisely with the cubic double field theory upon imposing a gauge-fixing condition and integrating out the dilaton. The need for a gauge-fixing condition is natural in view of amplitude computations: The double copy only works in the Feynman, respectively, de Donder gauges. In fact, we have also verified by a Noether construction that the sum of the quadratic and cubic double copy actions (17) and (26) cannot be gauge invariant. Thus, at best the above cubic piece (26) is related to a gauge-fixed form of double field theory.

It turns out to be convenient to use the form of double field theory originally derived from closed string field theory (SFT) [15]. We will not need any detailed technical background of SFT, but it is instructive to write out the string field truncated to the relevant states. The string field $\Psi$ takes values in the first-quantized Hilbert space and reads

$$
\begin{aligned}
|\Psi\rangle= & \int d K\left(-\frac{1}{2} e_{\mu \bar{\nu}}(K) \alpha_{-1}^{\mu} \bar{\alpha}_{-1}^{\bar{\nu}} c_{1} \bar{c}_{1}\right. \\
& +\varphi(K) c_{1} c_{-1}+\bar{\varphi}(K) \bar{c}_{1} \bar{c}_{-1} \\
& \left.+i f_{\mu}(K) c_{0}^{+} c_{1} \alpha_{-1}^{\mu}+i \bar{f}_{\bar{\mu}}(K) c_{0}^{+} \bar{c}_{1} \bar{\alpha}_{-1}^{\bar{\mu}}\right)|\mathbf{0} ; K\rangle,
\end{aligned}
$$

where $\alpha_{m}^{\mu}$ and $\bar{\alpha}_{m}^{\bar{\mu}}$ are the familiar left- and right-moving oscillators of the first-quantized string, and the $c_{m}$ and $\bar{c}_{m}$ are ghosts, being part of a $b c$ ghost system with algebra $\left\{b_{m}, c_{m}\right\}=\delta_{m+n}$ and $c_{0}^{ \pm}=\frac{1}{2}\left(c_{0} \pm \bar{c}_{0}\right)$. The state $|\mathbf{0} ; K\rangle$ is constructed from the so-called $S L(2, \mathbb{C})$-invariant vacuum $|\mathbf{0}\rangle$ by the action of $\exp (i k \cdot x)$ and $\exp (i \bar{k} \cdot \bar{x})$ and obeys in particular $b_{0}|\mathbf{0} ; K\rangle=\bar{b}_{0}|\mathbf{0} ; K\rangle=0$ [15]; see [27] for a review. Closed SFT yields for this truncated string field an action that reads to cubic order in position space [see equation (3.3) in [15] ]

$$
S_{\mathrm{SFT}}=\int d^{D} x d^{D} \bar{x}\left[\mathcal{L}^{(2)}+\mathcal{L}_{\mathrm{DC}}^{(3)}+\mathcal{L}_{\text {extra }}^{(3)}\right]
$$

where the superscripts denote the power of fields, and the various terms are defined as follows.

The quadratic action is given by

$$
\begin{aligned}
\mathcal{L}^{(2)}= & \frac{1}{4} e_{\mu \bar{\mu}} \square e^{\mu \bar{\mu}}+2 \bar{\varphi} \square \varphi-f_{\mu} f^{\mu}-\bar{f}_{\bar{\mu}} \bar{f}^{\bar{\mu}} \\
& -f^{\mu}\left(\bar{\partial}^{\bar{\nu}} e_{\mu \bar{\nu}}-2 \partial_{\mu} \bar{\varphi}\right)+\bar{f}^{\bar{\nu}}\left(\partial^{\mu} e_{\mu \bar{\nu}}+2 \bar{\partial}_{\bar{\nu}} \varphi\right) .
\end{aligned}
$$

We observe that $f_{\mu}$ and $\bar{f}_{\bar{\mu}}$ are auxiliary and can hence be integrated out algebraically. Doing so we recover the 
quadratic double field theory action in the form (17), with $\phi \equiv \varphi-\bar{\varphi}$ and the combination $\varphi+\bar{\varphi}$ dropping out and hence being pure gauge. The Lagrangian $\mathcal{L}_{\mathrm{DC}}^{(3)}$ precisely agrees with (26) obtained from the double copy construction. Thus, the complete double field theory action to cubic order differs from the double copy construction by $\mathcal{L}_{\text {extra }}^{(3)}$, which is given by

$$
\begin{aligned}
\mathcal{L}_{\text {extra }}^{(3)}= & \frac{1}{2} e_{\mu \bar{\nu}} f^{\mu} \bar{f}^{\bar{\nu}}-\frac{1}{2} f_{\mu} f^{\mu} \bar{\varphi}+\frac{1}{2} \bar{f}_{\bar{\nu}} \bar{f}^{\bar{\nu}} \varphi-\frac{1}{4} f^{\mu}\left(e_{\mu \bar{\nu}} \bar{\partial}^{\bar{\nu}} \bar{\varphi}+\bar{\partial}^{\bar{\nu}}\left(e_{\mu \bar{\nu}} \bar{\varphi}\right)\right)-\frac{1}{4} \bar{f}^{\bar{\nu}}\left(e_{\mu \bar{\nu}} \partial^{\mu} \varphi+\partial^{\mu}\left(e_{\mu \bar{\nu}} \varphi\right)\right) \\
& +\frac{1}{4} f^{\mu}\left(\bar{\varphi} \partial_{\mu} \varphi-\varphi \partial_{\mu} \bar{\varphi}\right)+\frac{1}{4} \bar{f}^{\bar{\nu}}\left(\bar{\varphi} \bar{\partial}_{\bar{\nu}} \varphi-\varphi \bar{\partial}_{\bar{\nu}} \bar{\varphi}\right)-\frac{1}{8} e_{\mu \bar{\nu}}\left(\bar{\varphi} \partial^{\mu} \bar{\partial}^{\bar{\nu}} \varphi+\varphi \partial^{\mu} \bar{\partial}^{\bar{\nu}} \bar{\varphi}-\partial^{\mu} \varphi \bar{\partial}^{\bar{\nu}} \bar{\varphi}-\bar{\partial}^{\bar{\nu}} \varphi \partial^{\mu} \bar{\varphi}\right)
\end{aligned}
$$

Upon integrating out $f$ and $\bar{f}$ including these cubic terms one recovers the standard cubic double field theory action of [15], up to field redefinitions. However, in the following it will be easier to stay in the first-order formulation and impose the gauge-fixing condition:

$$
f_{\mu}=\bar{f}_{\bar{\mu}}=0 .
$$

In principle, this leaves residual gauge transformations with $\lambda_{\mu}=\partial_{\mu} \chi$ and $\bar{\lambda}_{\bar{\mu}}=-\bar{\partial}_{\bar{\mu}} \chi$, but these are "trivial gauge parameters" whose action on fields vanishes. The above gauge condition is actually implied by a well-known gauge condition for SFT, known as Siegel gauge:

$$
b_{0}^{+}|\Psi\rangle=0,
$$

where $b_{0}^{+}=b_{0}+\bar{b}_{0}$. Indeed, acting with $b_{0}^{+}$on (27) and using $b_{0}^{+}|\mathbf{0} ; K\rangle=0$ one finds that the terms in the first and second line are annihilated, while the terms in the third line, using $\left\{b_{0}^{+}, c_{0}^{+}\right\}=1$, give a nonvanishing contribution. The Siegel gauge condition thus precisely amounts to setting $f_{\mu}=\bar{f}_{\bar{\mu}}=0$. One may also verify that setting $x=\bar{x}$, replacing $f$ and $\bar{f}$ by their lowest-order on-shell values, and setting the scalar dilaton to zero, this gauge condition implies the familiar de Donder gauge $\partial^{\mu} h_{\mu \nu}-\frac{1}{2} \partial_{\nu} h=0$.

Using the gauge condition (31) in (28) one obtains

$$
\begin{aligned}
S_{\mathrm{SFT}}= & \int d^{D} x d^{D} \bar{x}\left[\frac{1}{4} e_{\mu \bar{\mu}} \square e^{\mu \bar{\mu}}+2 \bar{\varphi} \square \varphi+\mathcal{L}_{\mathrm{DC}}^{(3)}\right. \\
& \left.-\frac{1}{8} e_{\mu \bar{\nu}}\left(\bar{\varphi} \partial^{\mu} \bar{\partial}^{\bar{\nu}} \varphi+\varphi \partial^{\mu} \bar{\partial}^{\bar{\nu}} \bar{\varphi}-\partial^{\mu} \varphi \bar{\partial}^{\bar{\nu}} \bar{\varphi}-\bar{\partial}^{\bar{\nu}} \varphi \partial^{\mu} \bar{\varphi}\right)\right] .
\end{aligned}
$$

For the quadratic theory we had to integrate out the dilaton $\phi \equiv \varphi-\bar{\varphi}$ from double field theory in order to show that it equals the double copy of Yang-Mills. A subtlety at this stage is that, after picking Siegel gauge, it is no longer true that only the combination $\phi \equiv \varphi-\bar{\varphi}$ enters the action. Thus, we have to integrate out the pair of fields $(\varphi, \bar{\varphi})$. Since these enter the action (33) only quadratically, at tree level integrating them out just amounts to setting $\varphi=\bar{\varphi}=0 .{ }^{3}$ Alternatively, we could integrate out $\varphi$ and $\bar{\varphi}$ from the full action (28) before gauge fixing, which owing to the linear couplings in (29) yields $\varphi=$ $\square^{-1}\left(\partial_{\mu} f^{\mu}\right)+\cdots$, etc., hence leading to a nonlocal action. But since these nonlocal terms involve $f$ and $\bar{f}$ they disappear in the gauge (31), and so the two operations of fixing a gauge and integrating out $\varphi, \bar{\varphi}$ commute. Either way, cubic double field theory in Siegel gauge precisely coincides with the double copy of Yang-Mills theory upon integrating out the scalar fields.

\section{SUMMARY AND OUTLOOK}

In this paper we have given the arguably most direct double copy prescription for the Yang-Mills action to cubic order and shown that it yields double field theory to this order (in which the duality-invariant dilaton has been integrated out). Remarkably, at quadratic order this relation holds at the level of gauge-invariant actions, i.e., without the need to impose a gauge condition or to introduce extra fields. While the match of the cubic terms does require gauge fixing, this gauge condition is well known in closed string field theory, from which double field theory was originally derived, as the Siegel gauge.

The question is whether, and if so how, this relation between Yang-Mills theory and double field theory extends to higher order in fields. The quartic part of the Yang-Mills Lagrangian (1) can be written as

$$
\mathcal{L}_{\mathrm{YM}}^{(4)}=-\frac{1}{4} g_{\mathrm{YM}}^{2} \kappa^{a b} f_{a c d} f_{b e f} A^{\mu c} A^{\nu d} A_{\mu}{ }^{e} A_{\nu}{ }^{f} .
$$

One subtlety in giving a double copy prescription for the quartic vertex directly is that although with (9) and (24) we have given double copy substitution rules for $\kappa_{a b}$ and $f_{a b c}$, the quartic vertex in the above form also requires the inverse $\kappa^{a b}$ of the Cartan-Killing metric, but $\bar{\Pi}^{\bar{\mu} \bar{\nu}}$ is not invertible. Nevertheless, a natural proposal for the double copy is a quartic Lagrangian in momentum space of the structural form

\footnotetext{
${ }^{3}$ Indeed, there are no tree-level diagrams for only external $e_{\mu \bar{\mu}}$ states that involve these fields and hence setting them to zero is the correct procedure of integrating them out at tree level.
} 


$$
\begin{aligned}
\mathcal{L}_{\mathrm{DC}}^{(4)} \propto & \eta^{\bar{\tau} \bar{\kappa}} \bar{\Pi}_{\bar{\tau} \bar{\mu} \bar{\nu}} \bar{\Pi}_{\bar{\kappa} \bar{\rho} \bar{\sigma}} e^{\mu \bar{\mu}} e^{\nu \bar{\nu}} e_{\mu}^{\bar{\rho}} e_{\nu}^{\bar{\sigma}} \\
& +\eta^{\tau \kappa} \Pi_{\tau \mu \nu} \Pi_{\kappa \rho \sigma} e^{\mu \bar{\mu}} e^{\nu \bar{\nu}} e^{\rho}{ }_{\bar{\mu}} e^{\sigma}{ }_{\bar{\nu}}
\end{aligned}
$$

which would give rise to a two-derivative action. As it stands, this proposal is incomplete since each of the $\Pi^{\mu \nu \rho}$ depends on three momenta [cf. (23)], while in the above terms there are only four $e$ fields, but at least structurally one can see that such an action reproduces many terms of the quartic double field theory, see [22].

It is clear that new ingredients are needed in order to realize double copy at the level of actions to all orders in fields. In particular, the perturbative expansion of double field theory is nonpolynomial and so a match with the Yang-Mills action, which is quartic, requires a suitable reformulation of both theories. Indeed, double copy suggests to bring both actions to cubic form upon introducing auxiliary fields, see e.g., [5], and more generally one may expect additional fields in order to realize the double copy in a manner that is off shell and fully gauge invariant. This may also provide a new perspective on the important open problem, alluded to in the Introduction, of constructing a weakly constrained double field theory beyond cubic order.
While in principle such a theory can be derived from the full closed string field theory by integrating out all massive string modes that do not belong to the double field theory sector [27,28], it would be greatly beneficial to have an efficient formulation of this theory as a double copied Yang-Mills action.

It would also be interesting to understand whether the web of double copy constructible theories, i.e., including various amounts of supersymmetries, gauged gravity theories and also nongravitational theories, may be connected to double field theory.

\section{ACKNOWLEDGMENTS}

We have greatly benefited from discussions with Roberto Bonezzi who in particular pointed out the relevance of the Siegel gauge. This work is supported by the Deutsche Forschungsgemeinschaft (DFG, German Research Foundation)-Project No. 417533893/ GRK2575 "Rethinking Quantum Field Theory" and by the European Research Council (ERC) under the European Union's Horizon 2020 research and innovation programme (Grant Agreement No. 771862).
[1] Z. Bern, J. J. M. Carrasco, and H. Johansson, New relations for gauge-theory amplitudes, Phys. Rev. D 78, 085011 (2008).

[2] Z. Bern, J. J. M. Carrasco, and H. Johansson, Perturbative Quantum Gravity as a Double Copy of Gauge Theory, Phys. Rev. Lett. 105, 061602 (2010).

[3] Z. Bern, J. J. Carrasco, M. Chiodaroli, H. Johansson, and R. Roiban, The duality between color and kinematics and its applications, arXiv:1909.01358.

[4] C. Wood, How gravity is a double copy of other forces, www.quantamagazine.org (2021).

[5] Z. Bern, T. Dennen, Y.-t. Huang, and M. Kiermaier, Gravity as the square of gauge theory, Phys. Rev. D 82, 065003 (2010).

[6] C. R. Mafra, O. Schlotterer, and S. Stieberger, Explicit BCJ numerators from pure spinors, J. High Energy Phys. 07 (2011) 092; N. E. J. Bjerrum-Bohr, J. L. Bourjaily, P. H. Damgaard, and B. Feng, Manifesting color-kinematics duality in the scattering equation formalism, J. High Energy Phys. 09 (2016) 094; Y.-J. Du and F. Teng, BCJ numerators from reduced Pfaffian, J. High Energy Phys. 04 (2017) 033; E. Bridges and C. R. Mafra, Algorithmic construction of SYM multiparticle superfields in the BCJ gauge, J. High Energy Phys. 10 (2019) 022.

[7] L. Borsten, B. Jurčo, H. Kim, T. Macrelli, C. Saemann, and M. Wolf, Becchi-Rouet-Stora-Tyutin-Lagrangian Double Copy of Yang-Mills Theory, Phys. Rev. Lett. 126, 191601 (2021).
[8] L. Borsten, H. Kim, B. Jurčo, T. Macrelli, C. Saemann, and M. Wolf, Double copy from homotopy algebras, Fortschr. Phys. 69, 2100075 (2021).

[9] R. Monteiro, D. O'Connell, and C. D. White, Black holes and the double copy, J. High Energy Phys. 12 (2014) 056; A. Luna, R. Monteiro, D. O'Connell, and C. D. White, The classical double copy for Taub-NUT spacetime, Phys. Lett. B 750, 272 (2015); A. Luna, R. Monteiro, I. Nicholson, A. Ochirov, D. O'Connell, N. Westerberg, and C. D. White, Perturbative spacetimes from Yang-Mills theory, J. High Energy Phys. 04 (2017) 069; M. Carrillo-González, R. Penco, and M. Trodden, The classical double copy in maximally symmetric spacetimes, J. High Energy Phys. 04 (2018) 028; A. Guevara, B. Maybee, A. Ochirov, D. O'connell, and J. Vines, A worldsheet for Kerr, J. High Energy Phys. 03 (2021) 201; R. Monteiro, D. O'Connell, D. P. Veiga, and M. Sergola, Classical solutions and their double copy in split signature, J. High Energy Phys. 05 (2021) 268; C. Cheung and J. Mangan, Covariant color-kinematics duality, J. High Energy Phys. 11 (2021) 069.

[10] L. J. Dixon, Calculating scattering amplitudes efficiently, in Theoretical Advanced Study Institute in Elementary Particle Physics (TASI 95): QCD and Beyond (1996) [arXiv:hep-ph/ 9601359]; H. Elvang and Y.-t. Huang, Scattering Amplitudes, arXiv:1308.1697; J. M. Henn and J. C. Plefka, Scattering Amplitudes in Gauge Theories (Springer, Berlin, 2014), Vol. 883. 
[11] H. Nicolai, From Grassmann to maximal $(N=8)$ supergravity, Ann. Phys. (Amsterdam) 19, 150 (2010).

[12] Z. Bern and A. K. Grant, Perturbative gravity from QCD amplitudes, Phys. Lett. B 457, 23 (1999).

[13] M. Tolotti and S. Weinzierl, Construction of an effective Yang-Mills Lagrangian with manifest BCJ duality, J. High Energy Phys. 07 (2013) 111; A. Anastasiou, L. Borsten, M. J. Duff, S. Nagy, and M. Zoccali, Gravity as Gauge Theory Squared: A Ghost Story, Phys. Rev. Lett. 121, 211601 (2018); L. Borsten and S. Nagy, The pure BRST Einstein-Hilbert Lagrangian from the double-copy to cubic order, J. High Energy Phys. 07 (2020) 093; P. Ferrero and D. Francia, On the Lagrangian formulation of the double copy to cubic order, J. High Energy Phys. 02 (2021) 213; M. Beneke, P. Hager, and A. F. Sanfilippo, Double copy for Lagrangians at trilinear order, arXiv:2106.09054.

[14] W. Siegel, Superspace duality in low-energy superstrings, Phys. Rev. D 48, 2826 (1993).

[15] C. Hull and B. Zwiebach, Double field theory, J. High Energy Phys. 09 (2009) 099.

[16] C. Hull and B. Zwiebach, The gauge algebra of double field theory and Courant brackets, J. High Energy Phys. 09 (2009) 090.

[17] O. Hohm, C. Hull, and B. Zwiebach, Background independent action for double field theory, J. High Energy Phys. 07 (2010) 016.

[18] O. Hohm, C. Hull, and B. Zwiebach, Generalized metric formulation of double field theory, J. High Energy Phys. 08 (2010) 008.

[19] G. Aldazabal, D. Marques, and C. Nunez, Double field theory: A pedagogical review, Classical Quantum Gravity 30, 163001 (2013); D. S. Berman and D.C. Thompson,
Duality symmetric string and M-theory, Phys. Rep. 566, 1 (2015); O. Hohm, D. Lüst, and B. Zwiebach, The spacetime of double field theory: Review, remarks, and outlook, Fortschr. Phys. 61, 926 (2013).

[20] A. A. Tseytlin, Duality symmetric closed string theory and interacting chiral scalars, Nucl. Phys. B350, 395 (1991); T. Kugo and B. Zwiebach, Target space duality as a symmetry of string field theory, Prog. Theor. Phys. 87, 801 (1992); W. Siegel, Two vierbein formalism for string inspired axionic gravity, Phys. Rev. D 47, 5453 (1993).

[21] O. Hohm, On factorizations in perturbative quantum gravity, J. High Energy Phys. 04 (2011) 103.

[22] R. H. Boels and C. Horst, Perturbative quantum gravity in double field theory, J. High Energy Phys. 04 (2016) 120.

[23] C. Cheung and G. N. Remmen, Twofold symmetries of the pure gravity action, J. High Energy Phys. 01 (2017) 104; Hidden simplicity of the gravity action, J. High Energy Phys. 09 (2017) 002.

[24] K. Lee, Kerr-schild double field theory and classical double copy, J. High Energy Phys. 10 (2018) 027.

[25] K. Kim, K. Lee, R. Monteiro, I. Nicholson, and D. P. Veiga, The classical double copy of a point charge, J. High Energy Phys. 02 (2020) 046.

[26] D. S. Berman, K. Kim, and K. Lee, The classical double copy for M-theory from a Kerr-Schild ansatz for exceptional field theory, J. High Energy Phys. 04 (2021) 071.

[27] A. S. Arvanitakis, O. Hohm, C. Hull, and V. Lekeu, Homotopy transfer and effective field theory II: Strings and double field theory, arXiv:2106.08343.

[28] A. Sen, Wilsonian effective action of superstring theory, J. High Energy Phys. 01 (2017) 108. 